EPJ Web of Conferences 71, 00140 (2014)

DOI: 10.1051/epjconf/20147100140

(C) Owned by the authors, published by EDP Sciences, 2014

\title{
Foundation of Hydrodynamics of Strongly Interacting Systems
}

\author{
Cheuk-Yin Wong ${ }^{1, a}$ \\ ${ }^{1}$ Physics Division, Oak Ridge National laboratory, Oak Ridge, TN 37831, U.S.A.
}

\begin{abstract}
Hydrodynamics and quantum mechanics have many elements in common, as the density field and velocity fields are common variables that can be constructed in both descriptions. Starting with the Schrödinger equation and the Klein-Gordon for a single particle in hydrodynamical form, we examine the basic assumptions under which a quantum system of particles interacting through their mean fields can be described by hydrodynamics.
\end{abstract}

\section{Introduction}

Hydrodynamics has applications in many areas of physics, for both finite and infinite systems [1]-[17]. We are interested in hydrodynamics of quantum systems in which particles obey quantum mechanics, as for example, in

- an atomic nucleus as a liquid drop,

- a finite quark-gluon plasma,

- an assembly of hadrons at the end point of a nuclear collision,

- a droplet of Bose-Einstein condensate,

- an astrophysical object of nuclear matter or neutron matter.

It should be realized on the outset that hydrodynamics and quantum mechanics have many elements in common. For example, the density field, $n(\boldsymbol{r}, t)$, and the velocity field, $\boldsymbol{u}(\boldsymbol{r}, t)$, are common variables that can be constructed in both descriptions. There are correspondingly similar equations of motion for $n(\boldsymbol{r}, t)$ and $\boldsymbol{u}(\boldsymbol{r}, t)$. They also have elements that are different, as for example in the relation between the pressure and the density in classical hydrodynamics or between the pressure and the wave function amplitude in quantum mechanics. It is useful to compare and contrast the similarities and differences so that their properties can be better understood.

In the case of a nucleus with a large number of nucleons, the gross static property can be described by the classical liquid drop model $[1,2]$. There are however finite-size quantum shell effects that arise from the quantization of single-particle states and these quantum effects exert great influences on the static nuclear geometrical configurations at their local energy minima. The interplay of both the classical bulk liquid-drop behavior and the quantum single-particle effect has led to rich phenomena of many local geometrical configurations built on top of a general underlying liquid-drop background ("Funny Hills" as described in [18]).

ae-mail: wongc@ornl.gov

This is an Open Access article distributed under the terms of the Creative Commons Attribution License 2.0, which permits unrestricted use, distribution, and reproduction in any medium, provided the original work is properly cited. 
A many-particle quantum mechanical system consists of many particles which interact with other particles. Much of the dynamics can be understood by the the simple picture of single particles moving in the mean-field $V(\boldsymbol{r}, t)$ generated by the other particles. Quantum effects will be pronounced in a finite system that is characterized by discrete states with wave functions within a finite geometrical region. In dynamics involving such a finite system, as in the evolution of the dense overlap region in high-energy nuclear collisions, the finite quantum effects may be interest. It is therefore useful to examine to what extent quantum mechanics for the motion of a single particle may be a part of the foundation for a hydrodynamical description of a quantum many-body system.

\section{Schrödinger Equation in Hydrodynamical Form}

To examine the behavior of one of the particles, we can investigate the particle moving in the mean-field $V(\boldsymbol{r}, t)$ with the time-dependent Schrödinger equation

$$
i \hbar \frac{\partial}{\partial t} \psi(\boldsymbol{r}, t)=-\frac{\hbar^{2}}{2 m} \nabla^{2} \psi(\boldsymbol{r}, t)+V(\boldsymbol{r}, t) \psi(\boldsymbol{r}, t) .
$$

We follow Madelung [19] and Bohm [20] to write the wave function in the form

$$
\psi(\boldsymbol{r}, t)=\phi(\boldsymbol{r}, t) \exp \{i S(\boldsymbol{r}, t)-i \Omega(t)\}
$$

where $\phi(\boldsymbol{r}, t), S(\boldsymbol{r}, t)$, and $\Omega(t)$ are real functions. We construct $\psi^{*} i \hbar \partial_{t} \psi-\psi\left[i \hbar \partial_{t} \psi\right]^{*}$ and we get

$$
\partial_{t} \phi^{2}+\nabla \cdot\left(\phi^{2} \frac{\hbar \nabla S}{m}\right)=0
$$

Upon identifying $\phi^{2}$ as the density field, $\rho$, and $\hbar \nabla S / m$ as the velocity field, $\boldsymbol{u}$, the above is just is the equation of continuity for $\rho$ and $\boldsymbol{u}$. We construct next $\psi^{*} i \hbar \partial_{t} \psi+\psi\left[i \hbar \partial_{t} \psi\right]^{*}$ and we get

$$
\hbar \phi^{2}\left(-2 \partial_{t} S-4 \partial_{t} \Omega\right)=\left\{-\frac{\hbar^{2}}{2 m} 2\left[\phi \nabla^{2} \phi-2 \phi^{2}(\nabla S)^{2}\right]\right\}+2 \phi^{2} V .
$$

Dividing the above by $\phi^{2}$, taking the gradient $\nabla_{i}$, and multiplying by $\phi^{2}$, we obtain

$$
\hbar \partial_{t}\left[\phi^{2} \nabla_{i} S\right]+\sum_{j=1}^{3} \nabla_{j}\left[\frac{\hbar^{2} \phi^{2}}{m} \nabla_{i} S \nabla_{j} S\right]=-\frac{\hbar^{2}}{2 m} \phi^{2} \nabla_{i} \frac{\nabla^{2} \phi}{\phi}-\phi^{2} \nabla_{i} V .
$$

If we define the quantum stress tensor as [21]

$$
p_{i j}^{(q)}=-\frac{\hbar^{2}}{2 m} \phi \nabla_{i} \nabla_{j} \phi+\frac{\hbar^{2}}{2 m} \nabla_{i} \phi \nabla_{j} \phi
$$

then the time-dependent Schrödinger equation can be written in the form

$$
\hbar \partial_{t}\left[\phi^{2} \nabla_{i} S\right]+\sum_{j=1}^{3} \nabla_{j}\left[\frac{\hbar^{2} \phi^{2}}{m} \nabla_{i} S \nabla_{j} S\right]=-\sum_{j=1}^{3} \nabla_{j} p_{i j}^{(q)}-\phi^{2} V .
$$

This is just is the Schrödinger equation in hydrodynamical form,

$$
\partial_{t}\left[\rho u_{i}\right]+\sum_{j=1}^{3} \nabla_{j}\left[\rho u_{i} u_{j}\right]=-\frac{1}{m} \sum_{j=1}^{3} \nabla_{j} p_{i j}^{(q)}-\frac{\rho}{m} V .
$$

The quantum stress tensor can also be written in two other alternative forms [21],

$$
\begin{aligned}
& \text { (ii) } \quad p_{i j}^{(q)}=-\frac{\hbar^{2}}{4 m} \delta_{i j} \nabla^{2} \phi^{2}+\frac{\hbar^{2}}{m} \nabla_{i} \phi \nabla_{j} \phi, \\
& \text { or (iii) } \quad p_{i j}^{(q)}=+\frac{\hbar^{2}}{4 m} \delta_{i j} \nabla^{2} \phi^{2}-\frac{\hbar^{2}}{m} \phi \nabla_{i} \nabla_{j} \phi \text {. }
\end{aligned}
$$




\section{An example of the quantum stress tensor}

We consider a plain wave with wave function $\phi(x)=A \cos (k x+B)$. The quantum pressure is

$$
p_{x x}=A^{2} \frac{\hbar^{2}}{2 m} k^{2}
$$

which is a constant inside a spatially flat region, perpendicular to the direction of motion. It increases with the square of the wave number, $k^{2}$, and is proportional to the kinetic energy of the single-particle state.

We consider next an exponentially decaying wave function, $\phi(x)=B e^{-\kappa x}$. The quantum pressure is

$$
p_{x x}=-A^{2} \frac{\hbar^{2}}{2 m} \kappa^{2}+A^{2} \frac{\hbar^{2}}{2 m} \kappa^{2}=0
$$

The stress tensor is zero beyond the point of decay.

We can now examine a square well of the form $V(x)=V_{0} \Theta(a-|x|)$ with a geometrical width $a$. A symmetric single-particle state wave functions is

$$
\psi(x)=A \cos k x \Theta(a-|x|)+B e^{-\kappa(|x|-a)} \Theta(|x|-a) ; \quad k=\sqrt{2 \mu\left(E-V_{0}\right)} ; \quad \kappa=\sqrt{2 \mu E},
$$

where $k$ and $\kappa$ satisfies the eigenvalue equation, $k \tan k a=\kappa$. An antisymmetric wave function can be similarly written down by replacing $\cos k x$ by $\sin k x$.
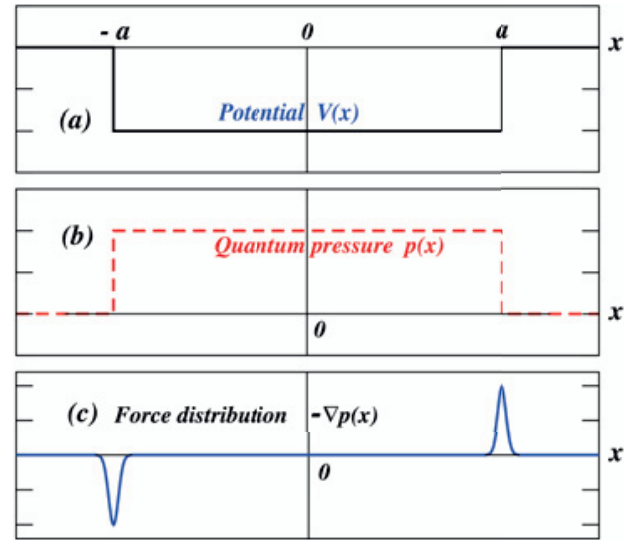

Fig. 1. The single-particle potential, quantum pressure, and force density in a square well.
For a wave function with $n$ numbers of nodes in a very deep potential well, the quantum pressure is

$$
p_{x x}=A^{2} \frac{\hbar^{2}}{2 m} k^{2} \sim \frac{\hbar^{2}}{m a^{3}} \frac{(2 n+1)^{2} \pi^{2}}{8},
$$

which reveals that the quantum stress tensor $p_{x x}(x)$ is constant inside the square well, proportional to the kinetic energy of the single particle measured relative to the bottom of the well. It depends sensitively on the geometrical width $a$ of the finite well, in contrast to a particle in an infinite medium without a boundary. The quantum stress tensor is zero outside the square well as depicted in Fig. 1 (b). As a consequence, the force distribution $-\nabla p(x)$ is sharply peak at the boundary and zero in the interior as in Fig. 1(c), directing outward.

In a self-assembled system with many single particles, the mean-field potential can be approximately represented by a square well. If the mean-field potential is suddenly weakened by the occurrence of a phase transition that alters the nature of the constituent interactions in the new phase, or if the external confining potential is suddenly removed, then hydrostatic equilibrium cannot be maintained and a hydrodynamical flow of a pressurized medium will then occur. 


\section{Hydrodynamical Description of a Nucleus}

We can examine a many-body system with strongly interacting constituents in the extended meanfield approximation [21-24] which is represented by a set of single-particle states $\psi_{a}$ in their mean field potential and a set of occupation numbers $n_{a}$.

The Schrödinger equation for the single-particle wave function $\psi_{a}$ in the mean field $V(\boldsymbol{r}, t)$ is

$$
i \hbar \frac{\partial}{\partial t} \psi_{a}(\boldsymbol{r}, t)=-\frac{\hbar^{2}}{2 m} \nabla^{2} \psi_{a}(\boldsymbol{r}, t)+V(\boldsymbol{r}, t) \psi_{a}(\boldsymbol{r}, t),
$$

and the occupation number $n_{a}$ obeys the master equation

$$
\frac{\partial n_{a}}{\partial t}=\sum_{234} \frac{2 \pi}{\hbar}\left|\left\langle a 2\left|v^{\prime}\right| 34 \mp 43\right\rangle\right|^{2} D\left(\epsilon_{a}+\epsilon_{2}-\epsilon_{3}+\epsilon_{4}\right)\left[n_{a} n_{2}\left(1 \mp n_{3}\right)\left(1 \mp n_{4}\right)-n_{3} n_{4}\left(1 \mp n_{a}\right)\left(1 \mp n_{2}\right)\right],
$$

where $D(x)$ is a delta-function type distribution with a width, the upper sign is for fermions and the lower sign is for bosons, $v^{\prime}$ is the residual interaction, and $\epsilon_{a}$ is the expectation value of the singleparticle Hamiltonian for the state $\psi_{a}$ that can be a slow function of time.

As the Schrödinger equation for a single-particle in an external field can be cast into a hydrodynamical form, we examine under what conditions a system of single particles interacting in their own mean fields can also be cast into a hydrodynamical form. We write

$$
\psi_{a}(\mathbf{r}, t)=\phi_{a}(\mathbf{r}, t) \exp \left\{-i S_{a}(\mathbf{r}, t)-i \Omega_{a}(t)\right\}
$$

We get

$$
\partial_{t} \phi_{a}^{2}+\nabla \cdot\left(\phi_{a}^{2} \frac{\hbar \nabla S_{a}}{m}\right)=0
$$

We multiple by $n_{a}$ and we get

$$
\partial_{t}\left[n_{a} \phi_{a}^{2}\right]+n_{a} \nabla \cdot\left[\phi_{a}^{2} \frac{\hbar \nabla S_{a}}{m}\right]=\phi_{a}^{2}\left[\partial_{t} n_{a}\right]
$$

Sum over $a$, we get,

$$
\partial_{t}\left[\sum_{a} n_{a} \phi_{a}^{2}\right]+\nabla \cdot\left[\sum_{a} n_{a} \phi_{a}^{2} \frac{\hbar \nabla S_{a}}{m}\right]=-\sum_{a} \phi_{a}^{2}\left[\partial_{t} n_{a}\right] .
$$

We can introduce the density matrix

$$
\mathcal{N}\left(\boldsymbol{r}, \boldsymbol{r}^{\prime} ; t\right)=\sum_{a} n_{a}(t) \psi_{a}^{*}(\boldsymbol{r}, t) \psi_{a}\left(\boldsymbol{r}^{\prime}, t\right)
$$

The diagonal element of the density matrix is the density field density field $\rho(\boldsymbol{r}, t)$

$$
\rho(\boldsymbol{r}, t)=\sum_{a} n_{a} \psi_{a}^{*}(\boldsymbol{r}, t) \psi_{a}(\boldsymbol{r}, t) .
$$

We can also introduce the velocity field $\boldsymbol{u}(\boldsymbol{r}, t)$

$$
\rho(\boldsymbol{r}, t) \boldsymbol{u}(\boldsymbol{r}, t)=\sum_{a} n_{a} \phi_{a}^{2}(\boldsymbol{r}, t) \frac{\hbar \nabla S_{a}(\boldsymbol{r}, t)}{m} .
$$


Equation (20) is then the equation of continuity

$$
\begin{aligned}
\partial_{t} \rho(\boldsymbol{r}, t)+\nabla \cdot[\rho(\boldsymbol{r}, t) \boldsymbol{u}(\boldsymbol{r}, t)]= & -\sum_{a} \phi_{a}^{2}\left[\partial_{t} n_{a}\right] \\
= & \frac{1}{4} \sum_{1234}\left[\phi_{1}^{2}+\phi_{2}^{2}-\phi_{3}^{2}-\phi_{4}^{2}\right] \frac{2 \pi}{\hbar}\left|\left\langle a 2\left|v^{\prime}\right| 34 \mp 43\right\rangle\right|^{2} \\
& \times D\left(\epsilon_{a}+\epsilon_{2}-\epsilon_{3}+\epsilon_{4}\right)\left[n_{a} n_{2}\left(1+\mp n_{3}\right)\left(1 \mp n_{4}\right)-n_{3} n_{4}\left(1 \mp n_{a}\right)\left(1 \mp n_{2}\right)\right],
\end{aligned}
$$

which contains an additional term on the right-hand side relating the scattering of a pair of particles with wave functions $\phi_{1}$ and $\phi_{2}$ non-locally into states of $\phi_{3}$ and $\phi_{4}$. Here, the factors $\left[\phi_{1}^{2}+\phi_{2}^{2}-\phi_{3}^{2}-\phi_{4}^{2}\right]$ and $\left[n_{a} n_{2}\left(1 \mp n_{3}\right)\left(1 \mp n_{4}\right)-n_{3} n_{4}\left(1 \mp n_{a}\right)\left(1 \mp n_{2}\right)\right]$ are antisymmetric in the exchange of 12 with 34 , so the above sum is not zero. However, the spatial integral of the right-hand side is zero. So, the total number of particles are conserved, even though a pair of particles can scatter into another pair non-locally.

For the single particle in state $a$, we can get the equation of motion for the probability current $\phi_{a}^{2} \nabla_{i} S_{a}[22]$

$$
\partial_{t}\left[\phi_{a}^{2} \nabla_{i} S_{a}\right]+\sum_{j=1}^{3} \nabla_{j}\left[\frac{\phi^{2}}{m} \nabla_{i} S_{a} \nabla_{j} S_{a}\right]=-\sum_{j=1}^{3} \nabla_{j} p_{i j}^{(q)}(a)-\phi_{a}^{2} V .
$$

We multiple by $n_{a}$ and sum over $a$, and we get the Euler equation for $n u_{i}$

$$
\frac{\partial n u_{i}}{\partial t}+\sum_{j=1}^{3} n u_{i} u_{j}=-\frac{1}{m} \sum_{j=1}^{3} \nabla_{j}\left(p_{i j}^{(q)}+p_{i j}^{(t)}+p_{i j}^{(v)}\right)-\sum_{a} \phi_{a}^{2} \nabla_{i} S_{a}\left[\partial_{t} n_{a}\right] .
$$

where

$$
\begin{aligned}
& p_{i j}^{(q)}=-\frac{\hbar^{2}}{4 m} \sum_{a} n_{a} \nabla^{2} \phi_{a}^{2} \delta_{i j}+\frac{\hbar^{2}}{m} \sum_{a} n_{a} \nabla_{i} \phi_{a} \nabla_{j} \phi_{a}, \\
& p_{i j}^{(t)}=\frac{\hbar^{2}}{m} \sum_{a} n_{a} \phi_{a}^{2}\left(\nabla_{i} S_{a}-m u^{i}\right)\left(\nabla_{j} S_{a}-m u^{j}\right), \\
& \nabla_{j} p_{i j}^{(v)}(\mathbf{r}, t)=\int d^{3} \mathbf{r}_{2}\left[\rho(\mathbf{r}, t) \rho\left(\mathbf{r}_{2}, t\right) \mp \mathcal{N}\left(\boldsymbol{r}, \boldsymbol{r}_{2} ; t\right) \mathcal{N}\left(\boldsymbol{r}_{2}, r ; t\right)\right] \nabla_{j} v\left(\mathbf{r}, \mathbf{r}_{2}\right),
\end{aligned}
$$

and $v\left(\mathbf{r}, \mathbf{r}_{2}\right)$ is the two-body interaction that generates the mean field. The last term in Eq. (26) arises from the change in current in the scattering of a pair of particles from state 12 to state 34 due to residual interactions $v^{\prime}$.

The equation of continuity (24) and the Euler equation (26) contain terms that depends on the change of the occupation probability $\left[\partial_{t} n_{a}\right]$. A finite system for which $n_{a}$ is quasi-stationary is said to be in thermal equilibrium, which can be attained when $\partial_{t} n_{a} \sim 0$. At thermal equilibrium, the occupation numbers obey

$$
n_{a}=\frac{1}{1 \mp \exp \left\{\left(\epsilon_{a}-\mu\right) / T\right\}},
$$

characterized by a Fermi energy $\mu$ and a temperature $T$ that can be a slow function of time. Here in the above equation, the upper sign is for fermions and the lwoer sign for boson. If a system 
with an initial occupation number $n_{a}$ that is far from the thermal equilibrium distribution, it will eventually reach thermal equilibrium after a thermal equilibriation time. When thermal equilibrium is reached, $\left[\partial_{t} n_{a}\right]=0$, and Eqs. (24) and (26) become the equations of motion in hydrodynamics. The requirement of thermal equilibrium is one of the basis for hydrodynamics.

After thermal equilibrium is reached, the total hydrodynamical pressure arises from many sources as indicated in Eqs. (26)-(29) : (i) the mean-field stress tensor $p_{i j}^{(v)}$ from the mutual interaction between fluid elements, (ii) the quantum stress tensor $p_{i j}^{(q)}$ from quantum effects, and (iii) the thermal stress tensor $p_{i j}^{(t)}$ from the deviation of the individual velocity fields from the local mean velocities. The stress tensor due to the mean-field interaction can also be given as

$$
\frac{\partial}{\partial x^{j}} p_{i j}^{(v)}(\mathbf{r}, t)=\rho(\mathbf{r}, t) \frac{\partial}{\partial x^{j}}\left(\frac{\partial W^{(v)}(\rho)}{\partial \rho}\right),
$$

where $W^{(v)}$ is the energy per particle arising from the mean-field interaction. The mean-field stress tensor $p_{i j}^{(v)}$ is given explicitly by

$$
p_{i j}^{(v)}=\left\{\rho \frac{\partial\left(W^{(v)} \rho\right)}{\partial \rho}-W^{(v)} \rho\right\} \delta_{i j} .
$$

For example, for a nucleus in which the nucleons interact with the Skyrm interaction, we have [22]

$$
p_{i j}^{(v)}=\frac{3}{8}\left(t_{0}+\frac{1}{3} t_{3} \rho\right) \rho^{2} \delta_{i j},
$$

where for the Skyrm I nucleon-nucleon interaction, $t_{0}=-1057 \mathrm{MeV} / \mathrm{fm}^{3}$ is the two-body interaction strength, and $t_{0}=+14463 \mathrm{MeV} / \mathrm{fm}^{6}$ is the three-body interaction strength.

The quantum stress tensor of a finite system with discrete states depends on the geometry of the system. Its magnitude varies inversely with the corresponding width of the system in that direction and needs to be examined on a case-by-case basis.

For a large fermion system with the neglect of the geometrical dependence, one can consider the Thomas-Fermi approximation of treating the single-particle states as a continuum and the quantum stress tensor for fermions is

$$
p_{i j}^{(q)}=\frac{\hbar^{2}}{5 m}\left(\frac{6 \pi^{2}}{4}\right)^{2 / 3} \rho^{5 / 3} \delta_{i j} .
$$

A nucleus is a strongly-coupled system. The quantum and mean-field stress tensors are the dominant component for the nuclear fluid at low and moderate temperatures. The thermal stress tensor $p_{i j}^{(t)}$ can take on different values, depending on the occupation numbers of the single-particle states that determines the degree of thermal equilibrium of the system. For a thermally equilibrated fermion system, the thermal stress tensor in the approximation of treating it as a local fermion gas is

$$
p_{i j}^{(t)}=\frac{\hbar^{2}}{5 m}\left(\frac{6 \pi^{2}}{4}\right)^{2 / 3} \rho^{5 / 3}\left[\frac{2 m k T}{\hbar^{2} m\left(6 \pi^{2} \rho / 4\right)^{2 / 3}}\right]^{2} \delta_{i j},
$$

which is small for low and moderate temperatures. Thus, when $\left|p_{i j}^{(q)}+p_{i j}^{(v)}\right| \gg p_{i j}^{(t)}$ in a strongly interacting system at low and moderate temperatures, there can be situations in which the system can behave quasi-hydrodynamically even though the state of the system has not yet reach thermal equilibrium, as is evidenced by the presence of nuclear collective vibrational and rotational states at low and moderate temperatures. In this case, the hydrodynamical state is maintained essentially by the quantum stress tensor and the strong mean-field stress tensor, and not by the thermal stress tensor. 


\section{Klein-Gordon equation in hydrodynamical form}

We wish to examine next a finite system of relativistic bosons as an assembly of single-particles obeying the Klein-Gordon equation and interacting with the mean-field generated by other particles. We would like to write the Klein-Gordon equation in hydrodynamical form in terms of the density and velocity fields. Here one encounters the well-known problem in Klein-Gordon equation that the naive probability density $\rho=2 \operatorname{Im}\left(\psi^{*} \partial_{t} \psi\right)$ constructed from the wave function $\psi$ is not necessarily a positive quantity. The presence of a negative probability density may appear to preclude its description in relativistic hydrodynamics.

A resolution of the pathology was provided by Feshbach and Villars [25] who reintroduced wave mechanical interpretation of the wave field $\psi$ by noting that the Klein-Gordon equation actually contains both particle and antiparticle degrees of freedom. The Klein-Gordon equation can be cast as a set of coupled time-dependent Schrödinger equations for the particle and antiparticle wave function components both with positive probability densities [25].

We shall follow Feshbach-Villar's method and consider a single-particle with a charge $e$ and a rest mass $m_{0}$ in a scalar field $\mathcal{S}$ and a gauge field $A_{\mu}$ [26]. The Klein-Gordon equation for the wave function of the single-particle is

$$
\left\{\left(i \hbar \partial_{t}-e A_{0}\right)^{2}-(-i \hbar \nabla-e \boldsymbol{A})^{2}-\left(m_{0}+\mathcal{S}\right)^{2}\right\} \Psi(r, t)=0
$$

where for brevity of notation, we shall abbreviate $m_{0}+\mathcal{S}$ by $M$. We consider the initial energy of the system to be $E$ which includes the rest mass $m_{0}$ so that $E$ is positive definite. If the external interactions are time-independent, then $E$ is a constant of motion. If the external fields are time-dependent, then $E$ changes with time and we need $E$ and $\partial_{t} E$ initially to start the time evolution. We can get $E>0$ as the expectation value of $i \hbar \partial_{t}$ and obtain $\partial_{t} E$ by an iterative procedure.

We introduce an auxiliary wave function $\Psi_{4}$,

$$
\left(i \hbar \partial_{t}-e A_{0}\right) \Psi=\left(E-e A_{0}\right) \Psi_{4} .
$$

Then the Klein-Gordon equation (36) becomes

$$
\left(i \hbar \partial_{t}-e A_{0}\right)^{2} \Psi=\Psi_{4}\left[i \hbar \partial_{t}\left(E-e A_{0}\right)\right]+\left(E-e A_{0}\right)\left(i \hbar \partial_{t}-e A_{0}\right) \Psi_{4},
$$

which allows us to obtain the equation for $\left(i \hbar \partial_{t}-e A_{0}\right) \Psi_{4}$ as

$$
\left(i \hbar \partial_{t}-e A_{0}\right) \Psi_{4}=\frac{1}{\left(E-e A_{0}\right)}\left\{\left\{(-i \hbar \nabla-e \boldsymbol{A})^{2}+M^{2}\right\} \Psi-\Psi_{4}\left[i \hbar \partial_{t}\left(E-e A_{0}\right)\right]\right\} .
$$

By the method of Feshbach and Villar [25], the Klein-Gordon equation (36) that is second order in time for $\Psi$ becomes a set of coupled equations (37) and (39) that are first order in time for $\Psi$ and $\Psi_{4}$. What remains is to turn the wave amplitudes $\Psi$ and $\Psi_{4}$ into particle and antiparticle wave function components. This can be accomplished by forming the sum and difference of Eqs. (37) and (39),

$$
\begin{aligned}
& \left(i \hbar \partial_{t}-e A_{0}\right)\left[\Psi+\Psi_{4}\right]=\frac{1}{\left(E-e A_{0}\right)}\left\{\left[(-i \hbar \nabla-e \boldsymbol{A})^{2}+M^{2}\right] \Psi+\left(E-e A_{0}\right)^{2} \Psi_{4}-\Psi_{4}\left[i \hbar \partial_{t}\left(E-e A_{0}\right)\right]\right\} \\
& \left(i \hbar \partial_{t}-e A_{0}\right)\left[\Psi-\Psi_{4}\right]=\frac{1}{\left(E-e A_{0}\right)}\left\{-\left[(-i \hbar \nabla-e \boldsymbol{A})^{2}+M^{2}\right] \Psi+\left(E-e A_{0}\right)^{2} \Psi_{4}+\Psi_{4}\left[i \hbar \partial_{t}\left(E-e A_{0}\right)\right]\right\}
\end{aligned}
$$

We identify the sum $\Psi+\Psi_{4}$ as the particle component $\psi_{e}$ of the wave function, and the difference $\Psi-\Psi_{4}$ as the antiparticle component $\psi_{\bar{e}}$ of the wave function by defining

$$
\psi_{e}=\frac{\Psi+\Psi_{4}}{2} \text { and } \psi_{\bar{e}}^{*}=\frac{\Psi-\Psi_{4}}{2}
$$


so that

$$
\Psi=\psi_{e}+\psi_{\bar{e}}^{*} \text { and } \Psi_{4}=\psi_{e}-\psi_{\bar{e}}^{*} .
$$

We then obtain a set of equations coupling the particle and antiparticle components of the wave functions [26],

$$
\begin{array}{r}
\left(i \hbar \partial_{t}-e A_{0}\right) \psi_{e}=\frac{1}{2\left(E-e A_{0}\right)}\left\{[(-i \hbar \nabla-e \boldsymbol{A}))^{2}+M^{2}+\left[\left(E-e A_{0}\right)^{2}-i \hbar \partial_{t}\left(E-e A_{0}\right)\right]\right] \psi_{e} \\
\left.\left.+[(-i \hbar \nabla-e \boldsymbol{A}))^{2}+M^{2}-\left[\left(E-e A_{0}\right)^{2}-i \hbar \partial_{t}\left(E-e A_{0}\right)\right]\right] \psi_{\bar{e}}^{*}\right\}, \\
\left(i \hbar \partial_{t}+e A_{0}\right) \psi_{\bar{e}}=\frac{1}{2\left(E-e A_{0}\right)}\left\{\left[(-i \hbar \nabla+e \boldsymbol{A})^{2}+M^{2}+\left[\left(E-e A_{0}\right)^{2}-i \hbar \partial_{t}\left(E-e A_{0}\right)\right]\right] \psi_{\bar{e}}\right. \\
\left.+\left[(-i \hbar \nabla+e \boldsymbol{A})^{2}+M^{2}-\left[\left(E-e A_{0}\right)^{2}-i \hbar \partial_{t}\left(E-e A_{0}\right)\right]\right] \psi_{e}^{*}\right\} .
\end{array}
$$

Thus, by the Feshbach-Villars method, the Klein-Gordon equation that is second-order in the time derivative can be decomposed into a set of coupled first-order Schrödinger equations containing particle wave function component $\psi_{e}$ and antiparticle wave function component $\psi_{\bar{e}}$. In these coupled equations, $\psi_{e}$ and $\psi_{\bar{e}}$ have positive norms, $\left|\psi_{e}\right|^{2}$ and $\left|\psi_{\bar{e}}\right|^{2}$, which can be interpreted as the densities of the probability fluid of particles and antiparticles respectively, as in hydrodynamics.

For simplicity of notation, we shall denote $\psi_{e}$ by $\psi_{+}$and $\psi_{\bar{e}}$ by $\psi_{-}$. A general solution of the KleinGordon equation for a particle with a charge $e$ contains a predominant particle component, $\psi_{e}=\psi_{+}$, and a small antiparticle component, $\psi_{\bar{e}}=\psi_{-}$. The above set of coupled equations (44) and (45) for a particle with a charge $e$ and a positive energy $E$ can be re-written compactly as

$$
\begin{array}{r}
\left(i \hbar \partial_{t} \mp e A^{0}\right) \psi_{ \pm}=\frac{1}{2\left(E-e A^{0}\right)}\left\{\left[\left(\frac{\hbar}{i} \nabla \mp e \boldsymbol{A}\right)^{2}+M^{2}+\left[\left(E-e A^{0}\right)^{2}-i \hbar \partial_{t}\left(E-e A^{0}\right)\right]\right] \psi_{ \pm}\right. \\
\left.+\left[\left(\frac{\hbar}{i} \nabla \mp e \boldsymbol{A}\right)^{2}+M^{2}-\left[\left(E-e A^{0}\right)^{2}-i \hbar \partial_{t}\left(E-e A^{0}\right)\right]\right] \psi_{\mp}^{*}\right\} .
\end{array}
$$

Similar to the above, the wave function for an antiparticle with a charge $\bar{e}=-e$ and a positive energy $E$ contains a predominant antiparticle component $\psi_{\bar{e}}\left(=\psi_{-}\right)$and a small particle component $\psi_{e}\left(=\psi_{+}\right)$ with positive norms, $\left|\psi_{ \pm}\right|^{2}$. The corresponding set of coupled equations for such an antiparticle can be obtained from the above Eq. (46) by changing $e \rightarrow-e$ and $\pm \rightarrow \mp$ to yield

$$
\begin{aligned}
\left(i \hbar \partial_{t} \pm e A^{0}\right) \psi_{\mp}=\frac{1}{2\left(E+e A^{0}\right)}\left\{\left[\left(\frac{\hbar}{i} \nabla \pm e \boldsymbol{A}\right)^{2}+M^{2}+\left[\left(E+e A^{0}\right)^{2}-i \hbar \partial_{t}\left(E+e A^{0}\right)\right]\right] \psi_{\mp}\right. \\
\left.+\left[\left(\frac{\hbar}{i} \nabla \pm e \boldsymbol{A}\right)^{2}+M^{2}-\left[\left(E+e A^{0}\right)^{2}-i \hbar \partial_{t}\left(E+e A^{0}\right)\right]\right] \psi_{ \pm}^{*}\right\} .
\end{aligned}
$$

Equation (46) for a predominantly particle state and Eq. (47) for a predominantly antiparticle state can be further rewritten in a more succinct form by introducing $e_{ \pm}= \pm e$ as [26]

$$
\begin{array}{r}
\left(i \hbar \partial_{t}-e_{ \pm} A^{0}\right) \psi_{ \pm}=\frac{1}{2\left(E-e_{ \pm} A^{0}\right)}\left\{\left[\left(\frac{\hbar}{i} \nabla-e_{ \pm} \boldsymbol{A}\right)^{2}+M^{2}+\left[\left(E-e_{ \pm} A^{0}\right)^{2}-i \hbar \partial_{t}\left(E-e_{ \pm} A^{0}\right)\right]\right] \psi_{ \pm}\right. \\
\left.+\left[\left(\frac{\hbar}{i} \nabla-e_{ \pm} \boldsymbol{A}\right)^{2}+M^{2}-\left[\left(E-e_{ \pm} A^{0}\right)^{2}-i \hbar \partial_{t}\left(E-e_{ \pm} A^{0}\right)\right]\right] \psi_{\mp}^{*}\right\},
\end{array}
$$

with $e_{+}=e$ for a predominantly particle state with a large $\psi_{+}$component, and $e_{+}=-e$ for a predominantly antiparticle state with a large $\psi_{-}$component. 
The second term inside the curly bracket on the right-hand side of the equation (48) represents the particle-antiparticle coupling and pair production. It involves essentially the difference between of $E^{2}$ and $\mathbf{p}^{2}+\left(m_{0}+\mathcal{S}\right)^{2}$ that is quite small when the strength of the interaction relative to the energy (or rest mass) of the particle is small.

\section{Equations of continuity for fluids of particles and antiparticles}

To see how the probability fluids of a particle behave in space and time, we consider the set of coupled equation (48) for the two components, $\psi_{ \pm}$, in terms of their amplitudes and phase functions,

$$
\psi_{ \pm}(\mathbf{r}, t)=\phi_{ \pm}(\mathbf{r}, t) e^{i S_{ \pm}(\mathbf{r}, t)-i \Omega_{ \pm}(t)} .
$$

We construct $\psi_{ \pm}^{*} \times(48)-\psi_{ \pm} \times(48)^{*}$. After some manipulations, we find

$$
\begin{aligned}
& \partial_{t}\left[\left(E-e_{ \pm} A^{0}\right) \phi_{ \pm}^{2}\right]+\nabla \cdot\left[\phi_{ \pm}^{2}\left(\nabla S_{ \pm}-e_{ \pm} \mathbf{A}\right)\right]=X_{ \pm}, \\
& \text {where } \quad \begin{aligned}
2 X_{ \pm}= & \left\{\chi_{ \pm}^{*}\left(\frac{\hbar}{i} \nabla-e_{ \pm} \mathbf{A}\right)^{2} \chi_{\mp}^{*}-\chi_{ \pm}\left(\frac{\hbar}{-i} \nabla-e_{ \pm} \mathbf{A}\right)^{2} \chi_{\mp}\right\} \\
& +\left[\left(m_{0}+\mathcal{S}\right)^{2}+\left(E-e_{ \pm} A\right)^{2}\right]\left(\chi_{ \pm}^{*} \chi_{\mp}^{*}-\chi_{ \pm} \chi_{\mp}\right)+\left[i \hbar \partial_{t}\left(E-e_{ \pm} A^{0}\right)\right]\left(\chi_{ \pm}^{*} \chi_{\mp}^{*}+\chi_{ \pm} \chi_{\mp}\right) .
\end{aligned}
\end{aligned}
$$

The quantities $X_{+}$and $X_{-}$are not generally a full divergence. The total number of particles and antiparticles in the two components are not separately conserved due to the production of particle pairs. However, the difference of the particle number and antiparticle numbers of the two components satisfies the equation

$$
\partial_{t}\left[\left(E-e_{ \pm} A^{0}\right)\left(\phi_{+}^{2}-\phi_{-}^{2}\right)\right]+\nabla \cdot\left[\phi_{+}^{2}\left(\nabla S_{+}-e_{+} \mathbf{A}\right)\right]-\nabla \cdot\left[\phi_{-}^{2}\left(\nabla S_{-}-e_{-} \mathbf{A}\right)\right]=X_{+}-X_{-},
$$

where $X_{+}-X_{-}$is a complete divergence,

$$
X_{+}-X_{-}=-\nabla \cdot\left(\psi_{+}^{*} \nabla \psi_{-}^{*}-\psi_{-}^{*} \nabla \psi_{+}^{*}\right) / 2+\nabla \cdot\left(\psi_{+} \nabla \psi_{-}-\psi_{-} \nabla \psi_{+}\right) / 2-\nabla \cdot\left[e_{+} A^{0}\left(\psi_{+}^{*} \psi_{-}^{*}+\psi_{+} \psi_{-}\right) / i\right]
$$

Therefore, the quantity

$$
n_{\text {particle }}=\int d \mathbf{r} \frac{E-e_{ \pm} A^{0}}{m_{0}}\left(\phi_{+}^{2}-\phi_{-}^{2}\right)=\int d \mathbf{r} \frac{E-e_{ \pm} A^{0}}{m_{0}}\left(\left|\psi_{+}\right|^{2}-\left|\psi_{-}\right|^{2}\right)
$$

is a conserved quantity because the right-hand side of the equation (53) is a complete divergence. The additional number of particles produced is equal to the additional number of antiparticles produced. The equal increase in particle and antiparticle numbers associated with $X_{+}$and $X_{-}$represents the occurrence of particle-antiparticle pair production by the mean field. A single-particle solution with a $n_{\text {particle }}=n_{ \pm}= \pm 1$ is one in which $\left|\psi_{ \pm}\right|^{2} \gg\left|\psi_{\mp}\right|^{2}$ and can be normalized to be

$$
\int d^{3} r \frac{E-e_{ \pm} A^{0}}{m}\left[\left|\psi_{ \pm}\right|^{2}-\left|\psi_{\mp}\right|^{2}\right]=1 \text { for a particle state with } n_{\text {particle }}= \pm 1 \text {. }
$$

Next, to obtain the Euler equation, we construct $\psi_{ \pm}^{*} \times(48)+\psi_{ \pm} \times(48)^{*}$, and we get

$$
\begin{aligned}
\phi_{ \pm}^{2}\left(-2 \partial_{t}\right. & \left.S_{ \pm}-2 e_{ \pm} A^{0}\right) \\
= & \frac{1}{2\left(E-e_{ \pm} A^{0}\right)}\left\{-\left[2 \phi_{ \pm} \nabla^{2} \phi_{ \pm}-2 \phi_{ \pm}^{2}\left(\nabla S_{ \pm}-e_{ \pm} \mathbf{A}\right)^{2}\right]+\left[\left(m_{0}+\mathcal{S}\right)^{2}+\left(E-e_{ \pm} A^{0}\right)^{2}\right] 2 \phi_{ \pm}^{2}\right. \\
& +\psi_{ \pm}^{*}\left(\frac{\hbar}{i} \nabla-e_{ \pm} \mathbf{A}\right)^{2} \psi_{\mp}^{*}+\psi_{ \pm}\left(\frac{\hbar}{-i} \nabla-e_{ \pm} \mathbf{A}\right)^{2} \psi_{\mp}+\left[\left(m_{0}+\mathcal{S}\right)^{2}-\left(E-e_{ \pm} A\right)^{2}\right]\left(\psi_{ \pm}^{*} \psi_{\mp}^{*}+\psi_{ \pm} \psi_{\mp}\right) \\
& \left.+\left[i \hbar \partial_{t}\left(E-e_{ \pm} A^{0}\right)\right]\left(\psi_{ \pm}^{*} \psi_{\mp}^{*}-\psi_{ \pm} \psi_{\mp}\right)\right\} .
\end{aligned}
$$


The quantities on the right-hand sides of Eqs. (52) and Eq. (56) contain terms of binary products such as $\psi_{ \pm}^{*} \psi_{ \pm}^{*}$ and $\psi_{ \pm} \psi_{ \pm}$. According to Eq. (48), they contain time factors $e^{2 i \Omega(t)}$ with a time frequency greater than $2 m_{0} / \hbar$ and they represent zitterbewegung motion of the coupling between the particle and antiparticle density fields and the current fields. Zitterbewegung leads to pair production, but the time average of these $e^{2 i \Omega(t)}$ contributions over a long period of time gives

$$
\left\langle\psi_{ \pm}^{*} \psi_{ \pm}^{*}+\psi_{ \pm} \psi_{ \pm}\right\rangle_{T} \sim \frac{\hbar}{2 m_{0} T}
$$

If the dynamical time scale $T \gg 1 / 2 m_{0}$, then terms of the type $\psi_{ \pm}^{*} \psi_{ \pm}^{*}$ and $\psi_{ \pm} \psi_{ \pm}$in $X_{ \pm}$becomes negligible when averaged over the time period $T$.

A hydrodynamical description will be appropriate after the stage of active pair production has passed and the expansion of the system is now driven by a slowly varying external field (or a mean field), with a dynamical time scale $T$ much greater than $\hbar / 2 m_{0}$. It is this type of motion for which we wish to provide a hydrodynamical description. In that case, the contributions from pair production and zitterbewegung motion by the mean field averaged over the time scale for mean-field motion may be small and neglected. We get uncoupled equations of motion for two kinds of particles. This is equivalent to the case of a "simple fluid" in relativistic hydrodynamics, in which the chemical composition of the fluid either ceases to change [4] or changes according to the requirement of thermodynamic equilibrium.

\section{Euler equation in the approximation of no pair production}

Under the circumstance when the pair-production and zitterbewegung arising from the mean-field can be neglected, terms of $\psi_{ \pm}^{*} \psi_{ \pm}^{*}$ and $\psi_{ \pm} \psi_{ \pm}$in Eqs. (52) and (56) can be neglected. After dividing Eq. (56) by $-2 \phi_{ \pm}^{2}$, the equation for the phase function $S_{ \pm}$for this simplified case is

$$
\left(\partial_{t} S_{ \pm}+e_{ \pm} A^{0}\right)=\frac{1}{2\left(E-e_{ \pm} A^{0}\right)}\left\{\left[\left(\nabla^{2} \phi_{ \pm}\right) / \phi_{ \pm}-\left(\nabla S_{ \pm}-e_{ \pm} \mathbf{A}\right)^{2}\right]-\left(m_{0}+\mathcal{S}\right)^{2}-\left(E-e_{ \pm} A^{0}\right)^{2}\right\}
$$

For this case with suppressed pair production, $e_{ \pm}=n_{ \pm} e= \pm e$. We take the gradient $\nabla_{i}$ of the above for $i=1,2,3$, and multiply by $\phi_{ \pm}^{2}\left(E-e_{ \pm} A^{0}\right)$. We obtain

$$
\begin{aligned}
\left(E-e_{ \pm}\right. & \left.A^{0}\right) \phi_{ \pm}^{2} \partial_{t}\left(\nabla_{i} S_{ \pm}-e_{ \pm} A^{i}\right) \\
= & \left\{\phi_{ \pm}^{2} \nabla_{i}\left[\left(\nabla^{2} \phi_{ \pm}\right) / 2 \phi_{ \pm}\right]-\sum_{j=1}^{3} \phi_{ \pm}^{2}\left(\nabla_{j} S_{ \pm}-e_{ \pm} A^{j}\right) \nabla_{j}\left(\nabla_{i} S_{ \pm}-e_{ \pm} A^{i}\right)\right. \\
& \left.-\left(m_{0}+\mathcal{S}\right) \phi^{2} \nabla_{i} \mathcal{S}-\sum_{j=1}^{3} \phi_{ \pm}^{2}\left(\nabla_{j} S_{ \pm}-e_{ \pm} A^{j}\right) e_{ \pm} F^{i j}\right\}-\left(E-e_{ \pm} A^{0}\right) \phi_{ \pm}^{2} e_{ \pm} F^{0 i} \\
& \left.+\frac{e_{ \pm} \phi_{ \pm}^{2} \nabla_{i} A^{0}}{2\left(E-e_{ \pm} A^{0}\right)}\left\{\left(\nabla^{2} \phi_{ \pm}\right) / \phi_{ \pm}-\left(\nabla S_{ \pm}-e_{ \pm} \mathbf{A}\right)^{2}\right]-\left(m_{0}+\mathcal{S}\right)^{2}+\left(E-e_{ \pm} A^{0}\right)^{2}\right\} .
\end{aligned}
$$


Using the equation of continuity for this simplified case without pair production, we obtain

$$
\begin{aligned}
\partial_{t}\left[\frac{\left(E-e_{ \pm} A^{0}\right) \phi_{ \pm}^{2}\left(\nabla_{i} S_{ \pm}-e_{ \pm} A^{i}\right)}{m_{0}+\mathcal{S}}\right]+\sum_{j=1}^{3} \nabla_{j}\left[\frac{\phi_{ \pm}^{2}\left(\nabla_{j} S_{ \pm}-e_{ \pm} A^{j}\right)\left(\nabla_{i} S_{ \pm}-e_{ \pm} A^{i}\right)}{m_{0}+\mathcal{S}}\right] \\
=-\frac{m}{m_{0}+\mathcal{S}} \sum_{j=1}^{3} \nabla_{j} p_{i j}^{(q)}-\phi_{ \pm}^{2} \nabla_{i} \mathcal{S}+\frac{1}{m_{0}+\mathcal{S}}\left\{-\left(E-e_{ \pm} A^{0}\right) \phi_{ \pm}^{2} e_{ \pm} F^{0 i}-\sum_{j=1}^{3} \phi_{ \pm}^{2}\left(\nabla_{j} S_{ \pm}-e_{ \pm} A^{j}\right) e_{ \pm} F^{i j}\right\} \\
\left.\quad+\frac{e_{ \pm} \phi_{ \pm}^{2} \nabla_{i} A^{0}}{2\left(E-e_{ \pm} A^{0}\right)\left(m_{0}+\mathcal{S}\right)}\left\{\left(\nabla^{2} \phi_{ \pm}\right) / \phi_{ \pm}-\left(\nabla S_{ \pm}-e_{ \pm} \mathbf{A}\right)^{2}\right]-\left(m_{0}+\mathcal{S}\right)^{2}+\left(E-e_{ \pm} A^{0}\right)^{2}\right\} \\
\quad-\left(E-e_{ \pm} A^{0}\right) \phi_{ \pm}^{2}\left(\nabla_{i} S_{ \pm}-e_{ \pm} A^{i}\right) \frac{\partial_{t} \mathcal{S}}{\left(m_{0}+\mathcal{S}\right)^{2}}-\sum_{j=1}^{3}\left[\frac{\phi_{ \pm}^{2}\left(\nabla_{j} S_{ \pm}-e_{ \pm} A^{j}\right)\left(\nabla_{i} S_{ \pm}-e_{ \pm} A^{i}\right)}{m_{0}+\mathcal{S}}\right] \frac{\nabla_{j} \mathcal{S}}{\left(m_{0}+\mathcal{S}\right)^{2}} .
\end{aligned}
$$

We can identity the fluid energy density $\epsilon_{ \pm}$as

$$
\epsilon_{ \pm}=\left(m_{0}+\mathcal{S}\right) \phi_{ \pm}^{2}
$$

as it corresponds to the fluid energy density for the fluid element at rest. The fluid element is characterized by a relativistic 4 -velocity $u^{\mu}$. We can identify

$$
\begin{aligned}
& u_{ \pm}^{0}=\frac{E-e_{ \pm} A^{0}}{m_{0}+\mathcal{S}} \\
& u_{ \pm}^{i}=\frac{\nabla_{i} S_{ \pm}-e_{ \pm} A^{i}}{m_{0}+\mathcal{S}}, \quad \text { for } i=1,2,3 .
\end{aligned}
$$

We can then write an equation of motion for $\epsilon_{ \pm} u_{ \pm}^{0} u_{ \pm}^{i}[26]$,

$$
\begin{aligned}
\partial_{t}\left(\epsilon_{ \pm} u_{ \pm}^{0} u_{ \pm}^{i}\right)+\sum_{j=1}^{3} \nabla_{j} \epsilon_{ \pm} u_{ \pm}^{i} u_{ \pm}^{j}+\frac{m}{m_{0}+\mathcal{S}} \sum_{j=1}^{3} \nabla_{j} p_{ \pm i j}^{(q)} \\
=-\phi_{ \pm}^{2} \nabla_{i} \mathcal{S}+\frac{1}{m_{0}+\mathcal{S}}\left\{-\left(E-e_{ \pm} A^{0}\right) \phi_{ \pm}^{2} e_{ \pm} F^{0 i}-\sum_{j=1}^{3} \phi_{ \pm}^{2}\left(\nabla_{j} S_{ \pm}-e_{ \pm} A^{j}\right) e_{ \pm} F^{i j}\right\} \\
\left.+\frac{e_{ \pm} \phi_{ \pm}^{2} \nabla_{i} A^{0}}{2\left(E-e_{ \pm} A^{0}\right)\left(m_{0}+\mathcal{S}\right)}\left\{\left(\nabla^{2} \phi_{ \pm}\right) / \phi_{ \pm}-\left(\nabla S_{ \pm}-e_{ \pm} \mathbf{A}\right)^{2}\right]-\left(m_{0}+\mathcal{S}\right)^{2}+\left(E-e_{ \pm} A^{0}\right)^{2}\right\} \\
-\left(E-e_{ \pm} A^{0}\right) \phi_{ \pm}^{2}\left(\nabla_{i} S_{ \pm}-e_{ \pm} A^{i}\right) \frac{\partial_{t} \mathcal{S}}{\left(m_{0}+\mathcal{S}\right)^{2}}-\sum_{j=1}^{3}\left[\frac{\phi_{ \pm}^{2}\left(\nabla_{j} S_{ \pm}-e_{ \pm} A^{j}\right)\left(\nabla_{i} S_{ \pm}-e_{ \pm} A^{i}\right)}{m_{0}+\mathcal{S}}\right] \frac{\nabla_{j} \mathcal{S}}{\left(m_{0}+\mathcal{S}\right)^{2}}
\end{aligned}
$$

where $i, j=1,2,3$. This is the Klein-Gordon equation for the particle and antiparticle probability densities in hydrodynamical form. The first two terms on the left-hand side correspond to $\partial_{\mu} T_{ \pm}^{\mu i}$, with the energy momentum tensor of the probability fluid $T_{ \pm}^{\mu i}=\epsilon_{ \pm} u_{ \pm}^{\mu} u_{ \pm}^{i}$, for $\mu=0,1,2,3$. The third term on the left-hand side is the quantum stress tensor arising from the spatial variation of the amplitude of the single-particle wave function [21],

$$
p_{ \pm i j}^{(q)}=-\frac{\hbar^{2}}{4 m} \nabla^{2} \phi_{ \pm}^{2} \delta_{i j}+\frac{\hbar^{2}}{m} \nabla_{i} \phi_{ \pm} \nabla_{j} \phi_{ \pm} .
$$

Thus the dynamics of the probability fluid obeys an equation similar to the hydrodynamical equation, with forces on fluid elements arising from what one expects in classical considerations. The additional element is the presence of the quantum stress tensor $p_{i j}^{(q)}$ that is proportional to $\hbar^{2}$ and arises from the quantum nature of the fluid. 


\section{Relativistic many-body system in the mean-field and no pair production approximation}

A many-body system in the time-dependent mean-field approximation consists of a collection of independent particles moving in the self-consistent mean-field generated by all other particles [21, 22, 27]. Each single-particle state $\psi_{a v}$ is characterized by a state label $a$, particle type $v$, energy $e_{ \pm}$, and occupation number $n_{a v}$. Under the approximation of no active pair production by the mean field, the dynamics of the system is now described by two distinct interacting fluids of particles and antiparticles. We consider the case in which the mean-field potential arises from a scalar two-body interaction $v_{s}\left(\mathbf{r}_{1}, \mathbf{r}_{2}\right)$ and a time-like vector interaction $v_{0}\left(\mathbf{r}_{1}, \mathbf{r}_{2}\right)$. For simplicity, we further neglect the last three terms on the right-hand side of Eq. (63) which represent higher-order relativistic corrections. The equation of motion for the energy density $\epsilon_{a v}$ and velocity fields $u_{a v}^{i}$ for $i=1,2,3$ and $v= \pm$, in the single particle state $a$ and particle type $v$, is then

$$
\partial_{t}\left(\epsilon_{a v} u_{a v}^{0} u_{a v}^{i}\right)+\sum_{j=1}^{3} \nabla_{j} \epsilon_{a v} u_{a v}^{i} u_{a v}^{j}+\frac{m}{m_{0}+\mathcal{S}} \sum_{j=1}^{3} \nabla_{j} p_{(a v) i j}^{(q)}=-\phi_{a v}^{2} \nabla_{i} \mathcal{S}+\frac{E-e_{a v} A_{ \pm}^{0}}{m_{0}+\mathcal{S}} \phi_{a v}^{2} e_{a v} \frac{\partial A^{0}}{\partial x^{i}},
$$

where, in the frame with the fluid element at rest,

$$
\begin{aligned}
& \mathcal{S}(\mathbf{r}, t)=\int d^{3} \mathbf{r}_{2} \rho\left(\mathbf{r}_{2}, t\right) v_{s}\left(\mathbf{r}, \mathbf{r}_{2}\right), \\
& A^{0}(\mathbf{r}, t)=\int d^{3} \mathbf{r}_{2}\left\{\rho_{+}\left(\mathbf{r}_{2}, t\right) e_{+}+\rho_{-}\left(\mathbf{r}_{2}, t\right) e_{-}\right\} v_{0}\left(\mathbf{r}, \mathbf{r}_{2}\right), \\
& \rho_{v}=\sum_{a} n_{a v} \phi_{a v}^{2}, \quad \text { and } \rho=\rho_{+}+\rho_{-} .
\end{aligned}
$$

We consider a strongly interacting system in which the number of particles and antiparticles are equal so that $\rho_{+}\left(\mathbf{r}_{2}\right)=\rho_{-}\left(\mathbf{r}_{2}\right)$ and $\rho_{+}\left(\mathbf{r}_{2}\right) e_{+}+\rho_{-}\left(\mathbf{r}_{2}\right) e_{-}$is zero. Then the contribution from the second term on the right-hand side of Eq. (65) is zero. Multiplying Eq. (65) by $n_{a v}$ and summing over $\{a$, $v\}$, we get

$$
\begin{array}{r}
\partial_{t}\left(\sum_{a v} n_{a v} \epsilon_{a v} u_{a v}^{0} u_{a v}^{i}\right)+\sum_{j=1}^{3} \nabla_{j}\left(\sum_{a v} n_{a v} \epsilon_{a v} u_{a v}^{i} u_{a v}^{j}\right)+\frac{m}{m_{0}+\mathcal{S}} \sum_{j=1}^{3} \nabla_{j}\left(\sum_{a v} n_{a v} p_{(a v) i j}^{(q)}\right)+\left(\sum_{a v} n_{a v} \phi_{a v}^{2}\right) \nabla_{i} \mathcal{S} \\
=\sum_{a v} \epsilon_{a v} u_{a v}^{0} u_{a v}^{i}\left[\partial_{t} n_{a v}\right]
\end{array}
$$

We define the total energy density $\epsilon$ by

$$
\sum_{a v} n_{a v} \epsilon_{a v}=\epsilon
$$

and the average 4-velocity $u$ by

$$
u=\sum_{a v} n_{a v} \epsilon_{a v} u_{a v} / \epsilon
$$

We can introduce the thermal stress tensor $p_{i j}^{(t)}$ for $\{i, j\}=1,2,3$ as the correlation of the deviations of the single-particle velocity fields from the average

$$
\sum_{a v} n_{a v} \epsilon_{a v}\left(u_{a v}^{i}-u^{i}\right)\left(u_{a v}^{j}-u^{j}\right) \equiv p_{i j}^{(t)}
$$


For the case with the suppression of pair production, we obtained the Euler equation of motion for $\epsilon u^{0} u^{i}$

$$
\partial_{t}\left(\epsilon u^{0} u^{i}\right)+\sum_{j=1}^{3}\left\{\nabla_{j}\left(\epsilon u^{i} u^{j}+p_{i j}^{(t)}+p_{i j}^{(v)}\right)+\frac{m}{m_{0}+\mathcal{S}} \nabla_{j} p_{i j}^{(q)}\right\}=\sum_{a v} \epsilon_{a v} u_{a v}^{0} u_{a v}^{i}\left[\partial_{t} n_{a v}\right],
$$

where the total quantum stress tensor is

$$
p_{i j}^{(q)}=-\frac{\hbar^{2}}{4 m} \nabla^{2} \sum_{a v} n_{a v} \phi_{a v}^{2} \delta_{i j}+\frac{\hbar^{2}}{m} \sum_{a v} n_{a v} \nabla_{i} \phi_{a v} \nabla_{j} \phi_{a v},
$$

and the pressure due to the interaction $p_{i j}^{(v)}$ is

$$
\frac{\partial}{\partial x^{j}} p_{i j}^{(v)}(\mathbf{r}, t)=n(\mathbf{r}, t) \nabla_{i} \mathcal{S}(\mathbf{r}, t)=n(\mathbf{r}, t) \frac{\partial}{\partial x^{j}} \int d^{3} \mathbf{r}_{2} n\left(\mathbf{r}_{2}, t\right) v_{s}\left(\mathbf{r}, \mathbf{r}_{2}\right) .
$$

The Euler equation of motion (26) for $\epsilon u^{0} u^{i}$ contains the term that depends on the change of the occupation probability $\left[\partial_{t} n_{a v}\right]$. A finite system for which $n_{a v}$ is quasi-stationary is said to be in thermal equilibrium, which can be attained when $\partial_{t} n_{a v} \sim 0$. When thermal equilibrium is reached, Eq. (73) become the Euler equation in hydrodynamics. The requirement of thermal equilibrium is one of the basis for hydrodynamics.

The mean-field stress tensor $p_{i j}^{(v)}$ can also be given as

$$
p_{i j}^{(v)}=\left\{n \frac{\partial\left(W^{(v)} n\right)}{\partial n}-W^{(v)} n\right\} \delta_{i j}
$$

where $W^{(v)}$ is the energy per particle arising from the mean-field interaction.

The quantum stress tensor $p_{i j}^{(q)}$ depends on the amplitudes of the wave functions while the thermal stress tensor $p_{i j}^{(t)}$ depends on the phases of the wave functions and the deviation of the velocity fields from the mean velocities. They can take on different values, depending on the occupation numbers $n_{a v}$ of the single-particle states that determine the degree of thermal equilibrium of the system. The quantum stress tensor is less sensitive to the degree of thermalization as compared to the thermal stress tensor. In the time-dependent mean-field description, the motion of each particle state can be individually followed [27]. The occupation numbers $n_{a v}$ of the single-particle states will remain unchanged, if there are no additional residual interaction between the single particles due to residual interactions. When particle residual interactions are allowed as in the extended time-dependent meanfield approximation [21-24], the occupation numbers will change and will approach an equilibrium distribution as time proceeds.

As the example of a finite potential in Section 3 indicates, the quantum stress tensor depends on the wave functions which depends on the geometry of the finite system. In a system with anisotropic shapes as in the overlapping dense region in a high-energy collision, the initial geometrical shape will have important influences on the quantum stress tensor in different directions and need to be explored further. If the mean-field potential is weakened by the occurrence of a phase transition that alters the nature of the constituent interactions in the new phase, then a non-isotropic hydrodynamical flow of a pressurized medium will then occur.

\section{Summary and Discussions}

For dense systems with strongly interacting constituents, a reasonable description of the system can be formulated in terms of constituents moving in the strong mean field generated by all other particles. 
Passage from the quantum mechanics to the hydrodynamics requires further the assumption of a thermal equilibrium such that the description of the occupation numbers of single-particle states in terms of a temperature can be a reasonable concept. It requires furthermore the assumption of no active pair production by the mean field such that the density flow and the momentum flow can be treated for particles and antiparticles as different fluids. From such an analysis, we find that the probability density and the current of the system obey hydrodynamical equations with the stress tensor arising from many contributions. There is the quantum stress tensor that arises from quantum effects and wave functions, there is the thermal stress tensor that arises from the deviation of the single-particle velocity fields from the average velocity fields, and there is the mean-field stress tensor that arises from the mean-field interactions.

The importance of the three different contributions depend on the physical situations that are present in the system. For low temperature dynamics and finite systems with non-isotropic geometries for which the quantum effects and mean field effects are important, the dynamics of the stronglycoupled system will be influenced more by the quantum stress tensor and the mean-field stress tensor than the degree of thermalization. On the other hand, for very high temperatures for which the magnitude of the thermal stress tensor far exceeds the strengths of the mean-field interactions and the quantum pressure, the thermal stress tensor plays the dominant role and the magnitude of the thermal stress tensor will depend sensitively on the degree of thermalization of the system. In between these limits, one can envisage the transition from the quantum and mean field dominating strongly-coupled regime to the thermal pressure dominating weakly-coupled regime as temperature increases.

As both the Schrödinger equation and the Klein-Gordon equation for bosons can be cast into a hydrodynamical form, one may inquire whether the Dirac equation for fermions can also be written in hydrodynamical form. It is well known that the Dirac equation can be reduced to a Klein-Gordon equation, with additional terms involving the spin and particle-antiparticle degrees of freedom. For a Dirac particle in an external field we have

$$
\left\{\gamma^{v}\left(i \partial_{ \pm}-e A_{ \pm}\right)-\left(m_{0}+\mathcal{S}\right)\right\} \psi=0 .
$$

Upon multiplying this on the left by $\gamma^{v}\left(i \partial_{ \pm}-e A_{ \pm}\right)+\left(m_{0}+\mathcal{S}\right)$, we obtain

$$
\left\{\left(i \partial_{ \pm}-e A_{ \pm}\right)^{2}-\left(m_{0}+\mathcal{S}\right)^{2}-i \alpha \cdot e \mathbf{E}+\sigma \cdot e \mathbf{B}(\mathbf{r})-i\left[\gamma^{\nu} \partial_{ \pm} \mathcal{S}\right]\right\} \psi=0,
$$

which is the Klein-Gordon equation with additional interactions. Thus, the Dirac equation can be likewise cast into a hydrodynamical form, following the procedures outlined in the present discussions.

What we have discussed is only a theoretical framework that exhibits clearly the different sources of stress tensors. To study specifically the dynamics of the quark-gluon plasma for example, it will be necessary to investigation the specific nature of different constituents and their interactions in a caseby-case basis. Nevertheless, the general roles played by the different components of stress tensors can still be a useful reminder on the importance of the quantum and mean-field stress tensors in the strongly-coupled regime, at temperature just above the transition temperature $T_{c}$.

\section{Acknowledgment}

This research was supported in part by the Division of Nuclear Physics, U.S. Department of Energy, under Contract No. DE-AC05-00OR22725. 


\section{References}

[1] N. Bohr and J. A. Wheeler, Phys. Rev. 56, 426 (1939).

[2] D. L. Hill and J. A. Wheeler, Phys. Rev. 89, 1102 (1953).

[3] C. W. Misner, K. S. Thorne, and J. A. Wheeler, Gravitation, W. H. Freeman Publisher, 1973.

[4] L. D. Landau and E. M. Lifshitz, Fluid Mechanics, Pergamon Press, 1959,

[5] K. Huang, Statistical Mechanics, J. Wiley \& Sons, N.Y. 1963.

[6] E. P. Gross, Nuovo Cimento 20, 454 (1961); E. P. Gross, J. Math. Phys. 4, 195 (1963).

[7] L. P. Pitaevskii, Zh. Eksp. Teor. Fiz. 40646 (1961) [Sov.Phys.JETP 13, 451 (1961)].

[8] L. Pitaevskii and S. Stringari, Bose-Einstein Condensation, Oxford University Press, Oxford, 2003.

[9] L. D. Landau, Izv. Akad. Nauk SSSR 17, 51 (1953), S. Z. Belenkij and L. D. Landau, Usp. Fiz. Nauk 56, 309 (1955); Nuovo Cimento, Suppl. 3, 15 (1956).

[10] J. D. Bjorken, Phys. Rev. D27, 140 (1983).

[11] G. Baym, B. L. Friman, J.-P. Blaizot, M. Soyeur, and W. Czyz, Nucl. Phys. A407, 541 (1983).

[12] B. D. Serot and J. D. Walecka, The Relativistic Many-body Problem, Plenum Press, 1986. .

[13] D.D. Clayton, Principles of Stellar Evolution and Nucleosynthesis, McGraw-Hill, 1968;

[14] G.W. Collins II, The Fundamentals of Stellar Astrophysics, W. H. Freeman Publisher, 1989.

[15] C. Y. Wong, Introduction to High-Energy Heavy-Ion Collisions, World Scientific Publisher, 1994.

[16] L. P. Csernai, Introduction to Relativistic Heavy-Ion Collisions, Wiley, 1994.

[17] J. Y. Ollitrault, Phys. Rev. D 46, 229, (1992); J. Y. Ollitrault, Eur. J. Phys. 29, 275 (2008); D. H. Rischke and M. Gyulassy, Nucl. Phys. A608, 479 (1996); D. Teaney, Phys. Rev. C68, 034913 (2004); T. Hirano and Y. Nara, Nucl. Phys. A743, 395 (2004); P. F. Kolb and U. Heinz,nuclth/0305084(2003); P. Huovinen and P. V. Ruuskanen, Ann. Rev. Nucl. Par. Sci. (2006); C. Nonaka and B. A. Bass, Phys. Rev. C75, 014902 (2007); O. J. Socolowski, F. Grassi, Y. Hama, and T. Kodama, Phys. Rev. Lett. 93, 182903 (2004); W. N. Zhang, M. J. Efaaf, and C. Y. Wong, Phys. Rev. C70, 024903 (2004); T. Csorgo, F. Grassi, Y. Hama, and T. Kodama, Phys. Lett. 565, 107 (2003); T. Csorgo et al., Phys. Lett. B663, 306 (2008); C. Y. Wong, Phys. Rev. C78, 054902 (2008); R. Peschanski and E. N. Saridakis, Phys. Rev. C80, 024907 (2009).

[18] M. Brack, J. Damgaard, A.S. Jensen, H. C. Pauli, V. M. Strutinsky, and C. Y. Wong, Rev. Mod. Phys. 44, 320 (1972).

[19] E. Madelung, Z. Phys. 40, 332 (1926).

[20] D. Bohm, Phys. Rev. 85, 166 (1952) and Phys. Rev. 85, 180 (1952).

[21] C. Y. Wong, J. Math. Phys. 17, 1008 (1976).

[22] C. Y. Wong, J. A. Maruhn, and T. A. Welton, Nucl. Phys. A253, 469 (1975); C. Y. Wong, T. A. Welton, and J. A. Maruhn, Phys. Rev. C15, 1558 (1977); C. Y. Wong and J. A. McDonald, Phys. Rev. C16, 1196 (1977), C. Y. Wong, Phys. Rev. C17, 1832 (1978),C. Y. Wong and H. H. K. Tang, Phys. Rev. Lett. 40, 1070 (1978) ; C. Y. Wong and H. H. K. Tang, Phys. Rev. C20, 1419 (1979), C. Y. Wong, Phys. Rev. C 25, 1460 (1982).

[23] S. Ayik, Z. Phys. A 298, 83 (1980).

[24] D. Lacroix, P. Chomaz, S. Ayik, Nucl. Phys. A651, 369 (1999).

[25] H. Feshbach and F. Villars, Rev. Mod. Phys. 30, 24 (1958).

[26] C. Y. Wong, J. Math. Phys. 51, 112304 (2010).

[27] P. Bonche, S. Koonin, and J. W. Negele, Phys. Rev. C 13, 1226 (1976) . 